\title{
Diabetes in pregnancy and birth weight: Differential effects due to ethnicity
}

\author{
J Wilson, H Venkataraman, J Cheong, N Sukumar, S Seaton, K Khunti \& P Saravanan
}

\section{Introduction}

Macrosomia (birth weight $>4.5 \mathrm{~kg}$ ) is a well-recognised complication of maternal hyperglycaemia and is associated with poor maternal and foetal outcomes[1]. Little is known on how diabetes differentially affects birth weight across different ethnicities. We use a large hospital acquired dataset to explore the contribution of diabetes on birth weight in South Asian (SA) and White Caucasian (WC) populations living in the UK.

Table 1: Overview of birth weight predictors across ethnicity ${ }^{1}$

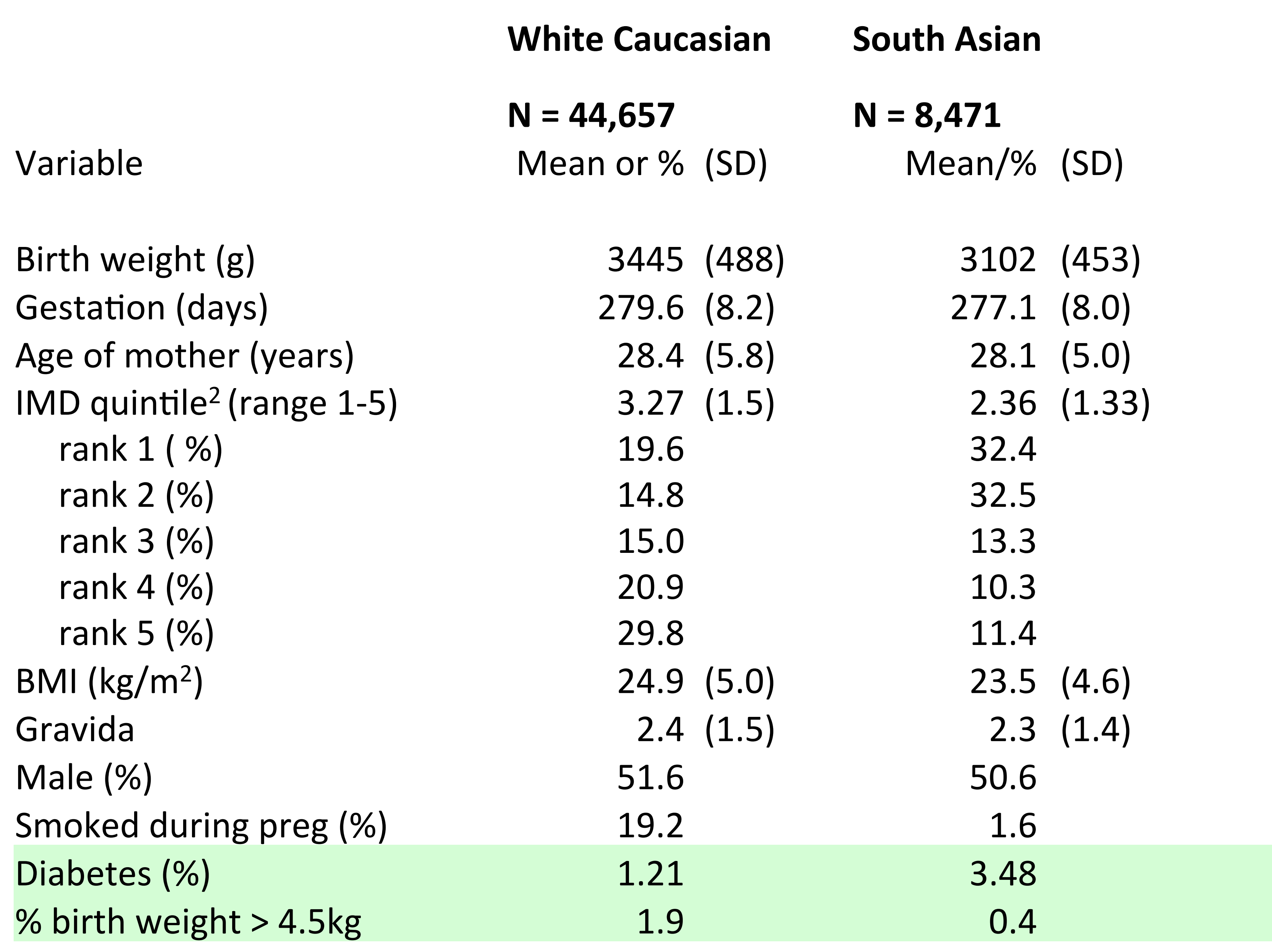

${ }^{1}$ All $p$ values for difference $<0.001$ except gravida $(p=0.04)$ and $\operatorname{sex}(p=0.08)$ ${ }^{2}$ Index of Multiple Deprivation rank $1=$ most deprived

\section{Methodology}

Data from 88,606 singleton births were analysed from the obstetric database maintained at University Hospitals of Leicester (1994-2006). Known predictors of birth weight (BW) were included in the model as shown in Table 1. After excluding missing or inaccurate data and restricting ethnicity to South Asian (SA) or White Caucasian (WC), 53,128 births had complete data of mother and baby. Postcode data is used to establish relative levels of deprivation using the Index of Multiple Deprivation[2]. Diabetes data includes both gestational and pre-gestational diabetes and is collected using local hospital methods.

Univariable and multivariable linear and logistic regression models are used to analyse predictors of BW in full-term births ( $\geq 37$ and $<42$ weeks). The interaction between ethnicity and diabetes is further scrutinised. Analysis is performed using Stata version 11[3].
Figure 1: distribution of birth weights by ethnicity and diabetes status

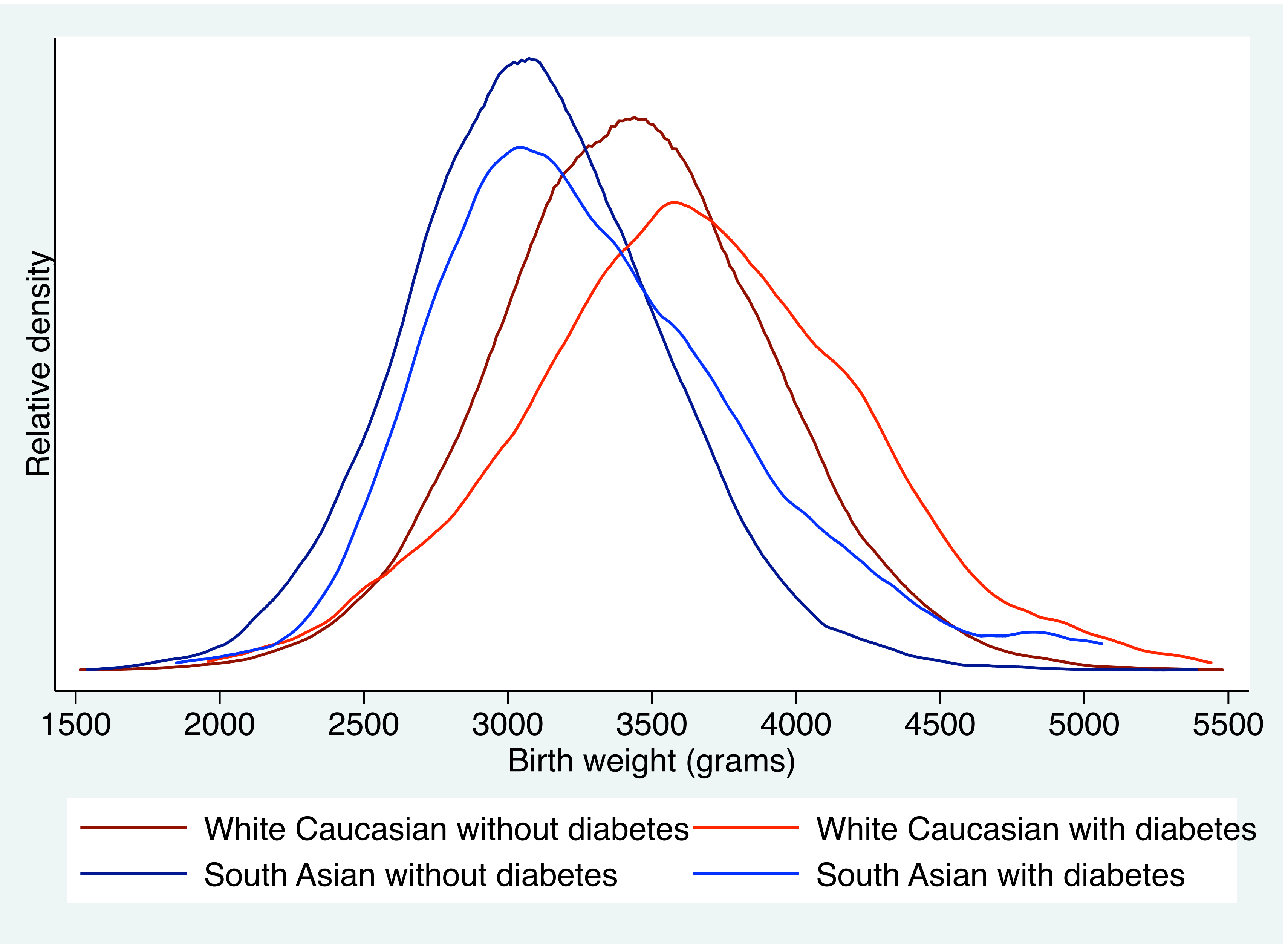

Results

Table 1 describes the BW predictors across SA and WC ethnicities. Figure 1 illustrates the unadjusted birth weight distributions by ethnicity and maternal diabetes status. The results of both linear and logistic regression models are shown in Table 2. We find all variables included in both the unadjusted and adjusted models to be significant predictors of BW.

Maternal diabetes increases BW by $276.3 \mathrm{~g}$ ( $\mathrm{Cl} 237.8$ to $296.8 \mathrm{~g}$ ) and the odds of macrosomia by 3.79 fold ( $\mathrm{Cl} 2.81$ to 5.81 ) across all ethnicities and after adjusting for known confounders. WC births are on average $272.2 \mathrm{~g}(\mathrm{Cl} 261.6$ to $282.7 \mathrm{~g}$ ) heavier and the odds of macrosomia are increased 4.55 fold $(\mathrm{Cl}$ 3.33 to 6.67) compared to SA births after correcting for confounders.

For WC mothers, maternal diabetes increases BW by an average of $293.2 \mathrm{~g}$ compared to $218.6 \mathrm{~g}$ for SA mothers (difference $74.6 \mathrm{~g}, \mathrm{Cl} 13.4$ to $135.8 \mathrm{~g}$, $p=0.017$ ). The odds of macrosomia in SA births are increased 12.39 fold with maternal diabetes, compared to a 5.20 fold increase for WC births (ratio of OR 2.38, $\mathrm{Cl} 1.05$ to $5.40, \mathrm{p}=0.037)$.

\section{Conclusions}

- After adjustment for known BW predictors we find WC births are on average $272 \mathrm{~g}$ heavier than SA births, indicating that the factors affecting interplay of ethnicity and BW are still largely unknown. This correlates with previous cross-sectional studies[4]. Whether this in turn affects long term metabolic risks seen in SA needs to be studied.

- Ethnicity significantly modulates the effect of diabetes on birth weight. The absolute increase in birth weight due to maternal diabetes is less in SA than WC births. The odds of macrosomia due to diabetes are increased more for SA compared to WC births; reflecting the lower absolute prevalence of macrosomia in SA births.

Table 2: Univariable (unadjusted) and multivariable (adjusted) predictors of BW. OR: odds ratio for macrosomia (BW > 4.5kg), Cl: confidence interval

\begin{tabular}{|c|c|c|c|c|c|c|}
\hline Predictor & Univariable coefficient /g (Cl) ${ }^{1}$ & Multivariable coefficient /g (Cl) ${ }^{2}$ & Univariable OR (Cl) & p value & Multivariable OR (Cl) & $p$ value \\
\hline Ethnicity (SA=1) & $-342.2(-353.4,-330.9)$ & $-272.2(-282.7,-261.6)$ & $0.22(0.15,0.30)$ & $<0.001$ & $0.26(0.18,0.37)$ & $<0.001$ \\
\hline Sex (Female=1) & $-128.1(-136.6,-119.7)$ & $-137.8(-145.0,-130.5)$ & $0.48(0.41,0.55)$ & $<0.001$ & $0.46(0.40,0.53)$ & $<0.001$ \\
\hline Gestation (days) & $23.6(23.2,24.1)$ & $22.9(22.5,23.4)$ & $1.09(1.08,1.10)$ & $<0.001$ & $1.10(1.09,1.11)$ & $<0.001$ \\
\hline Age of mother (yrs) & $8.0(7.2,8.8)$ & $1.9(1.1,2.6)$ & $1.04(1.03,1.06)$ & $<0.001$ & $1.02(1.01,1.04)$ & 0.001 \\
\hline $\mathrm{BMI}\left(\mathrm{m} / \mathrm{kg}^{2}\right)$ & $19.3(18.4,20.1)$ & $14.5(13.8,15.2)$ & $1.10(1.08,1.11)$ & $<0.001$ & $1.08(1.07,1.10)$ & $<0.001$ \\
\hline IMD rank 1 & 0.0 & 0.0 & 1.00 & & 1.00 & \\
\hline rank 2 & $-83.1(-94.2,-72.0)$ & $41.3(29.6,53.1)$ & $0.99(0.78,1.25)$ & 0.9 & $1.04(0.81,1.32)$ & 0.8 \\
\hline rank 3 & $16.9(4.9,28.9)$ & $67.0(54.4,79.5)$ & $1.27(1.01,1.61)$ & 0.04 & $1.14(0.89,1.46)$ & 0.3 \\
\hline rank 4 & $54.3(43.5,65.0)$ & $71.4(59.4,83.4)$ & $1.18(0.94,1.47)$ & 0.15 & $0.98(0.77,1.24)$ & 0.9 \\
\hline rank 5 & $114.2(104.6,123.7)$ & $106.5(95.0,118.0)$ & $1.56(1.28,1.90)$ & $<0.001$ & $1.33(1.07,1.65)$ & 0.01 \\
\hline Gravida & $21.0(18.2,23.9)$ & $25.4(22.7,28.1)$ & $1.12(1.08,1.17)$ & $<0.001$ & $1.10(1.06,1.15)$ & $<0.001$ \\
\hline Smoker & $-107.4(-118.8,-96.1)$ & $-140.3(-150.6,-130.0)$ & $0.59(0.48,0.74)$ & $<0.001$ & $0.56(0.45,0.70)$ & $<0.001$ \\
\hline Diabetes & $133.9(99.9,167.9)$ & $267.3(237.8,296.8)$ & $3.79(2.81,5.11)$ & $<0.001$ & $6.01(4.30,8.41)$ & $<0.001$ \\
\hline
\end{tabular}

